\title{
Study of Impact Erosion of Oil and Gas Flowlines with Micro-sized Sand Particles
}

\author{
Muhammad Y Naz ${ }^{1}$, Shaharin A Sulaiman ${ }^{2,}$, Noor I Ismail ${ }^{3}$, Muddasser Inayat $^{2}$, Shazia \\ Shukrullah $^{1}$, and $A b d u l$ Ghaffar $^{1}$ \\ ${ }^{1}$ Department of Physics, University of Agriculture, 38040 Faisalabad, Pakistan \\ ${ }^{2}$ Department of Mechanical Engineering, Universiti Teknologi PETRONAS, Malaysia \\ ${ }^{3}$ Department of Petroleum Engineering, Universiti Teknologi PETRONAS, Malaysia
}

\begin{abstract}
The oil and gas produced from the reservoirs of relatively low formation strength inevitably carry micro-sized sand particles, which adversely influence the flow-lines and other installations. The financial deliberations favor the choice of low-cost metals, in general, carbon steel as a flow-line material. Yet, there are many cases where carbon steel been substituted with a low-cost stainless steel. The corrosion resistance of the stainless-steel is well accepted. However, there is another type of deformation that is not well studied and understood is its erosion resistance. The objective of this study is to investigate the factors affecting the erosion of stainless steel placed in a dry sand stream of micro-size particles. The manipulated variables were sand size, impingement angle, gas flow velocity, and nozzle distance. The obtained results showed that the large particle size, high fluid-flow velocity and short nozzle distance would result in more severe erosion of the stainless-steel coupons.
\end{abstract}

\section{Introduction}

The recovery of crude oil from the reservoirs is a complex process, which may cause significant financial losses if not handled properly. During oil recovery from reservoir, the selection of equipment and network of pipes require economic considerations. The economic factor has determined the choice of low-cost metals, in general, the carbon steel as a flowline material [1]. However, there are situations where carbon steel does not give the required performance and the practice is then to employ a low cost stainless steel. An example of aggressive brine where carbon steel can fail is the brine containing a weak acid, such as acetic acid [2]. This is due to the enhancement in the carbon steel corrosion rate. For stainless steel, its corrosion rate has been extensively studied and the role of a passivating film, a few nanometers thick film of chromium inhibiting corrosion, is generally acceptable [3].

Erosion is another type of the deformation, which damages to the oil and gas facilities and equipment. When oil and gas are produced from the reservoirs of relatively low formation strength, sand detaches from the reservoir. Therefore, some of the sand is also produced along with fluids [2]. These micro-sized sand particles can cause erosion on the

\footnotetext{
* Corresponding author: shaharin@utp.edu.my
} 
pipelines and equipment, leading to production shutdown. According to Islam and Farhat [4], the cost of erosion attack to the global oil and gas industry has drawn significant attention amongst researchers in recent years. In fact, the studies conducted by Shirazi [5] and Wong et al. [6] had documented about the sand erosion in facilities and pipe systems for multiphase media. Islam and Farhat [4], discovered that higher particle velocity leads to more material removal. In addition, reduction in erosive wear is related to an increase in distance between erodent particles and target material. This is because of reduced influence of kinetic energy and gravitational force of the particle [7]. The objective of the present work was to elaborate the factors affecting the erosion of stainless-steel placed in a dry sand stream at different angles and impact velocities.

\section{Methodology}

A dry sieving technique was employed to obtain the desired sand size. It was done by using a series of meshes having gradually smaller screen sizes as they go down the stack. raw sand was passed through a series of screens with gradually decreasing mesh sizes. The screens were shaken mechanically to ease the seeping process. A fine particle size of $63 \mu \mathrm{m}$ and medium size of $150 \mu \mathrm{m}$ was obtained for sand impact erosion experiments. In erosion experiments, fine polished coupons of stainless steel were blasted with sand stream using a custom-built sand blasting equipment. Schematic of the setup is shown in Figure. 1.

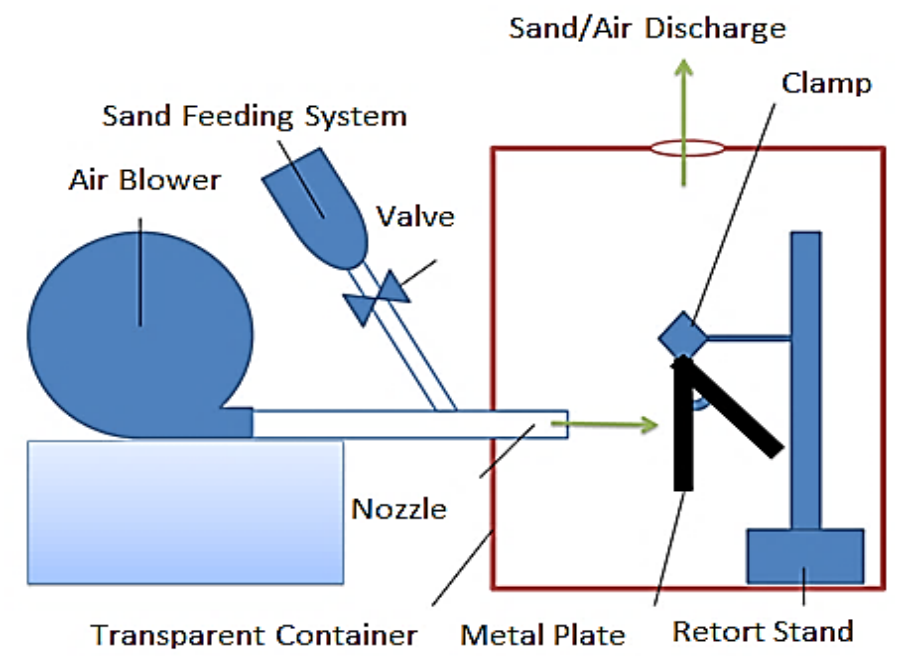

Fig. 1. Schematic of the experimental setup.

The stainless-steel coupons were used as representative surface of the flow-line facility. The sand particles were accelerated and impinged on the target surface through an air blower. The coupons were placed in metal chamber and bombarded with sand at different impact angles and velocities. Two particle sizes were considered in the presented work. The sand flowrate and bombardment time were fixed at $1 \mathrm{~kg} / \mathrm{h}$ and $1 \mathrm{~h}$, respectively. The erodent particles were classified into medium size sand $(150 \mu \mathrm{m})$ and fine size sand $(<63 \mu \mathrm{m})$. The coupons were bombarded with these sand sizes at two impact angles $\left(45^{\circ}\right.$ and $\left.90^{\circ}\right)$. The distance between coupon and nozzle was initially fixed at $10 \mathrm{~mm}$, which was changed to $20 \mathrm{~mm}$ at the later stage. The sand stream velocity was varied from $54 \mathrm{ft} / \mathrm{s}$ to $72 \mathrm{ft} / \mathrm{s}$. The effect of impact velocity, nozzle distance and impact angle on morphology of the eroded coupons was investigated using SEM and USPM techniques. 


\section{Results and discussion}

The sand blasting technique was used to investigate the severity of erosion of flow-lines with different sand sizes, using fine sand $(<63 \mu \mathrm{m})$ and medium-sized sand $(150 \mu \mathrm{m})$. The sand impact angle, sand velocity and target distance were changed to understand the basic erosion mechanism involved in the erosion process. Figure 2 shows SEM micrographs of untreated and treated stainless steel plates. The blank coupons were clean and revealed shiny texture. There were no marks or rough patches on the blank coupons. The severity of the sand blasting of the blank coupons was then easily understandable since there were no external damaging factors other than the sandblasted. The sand exposed coupons of stainless steel turned to dark and revealed the strong dependence of surface roughness on the process parameters, as shown in Figure 3. The impact velocity and impact angle significantly influenced the level of surface roughness. The sand stream, accelerated at high velocity, eroded the coupons more deeply than the slow-moving sand particles.
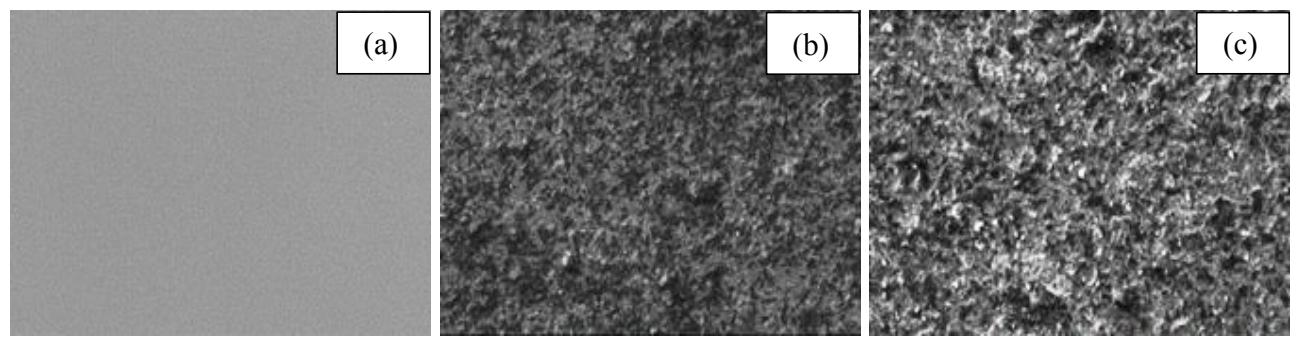

Fig. 2. 300 times magnified SEM micrographs of: (a) blank sampled, (b) eroded with fine sand, and (c) eroded with medium-sized sand.

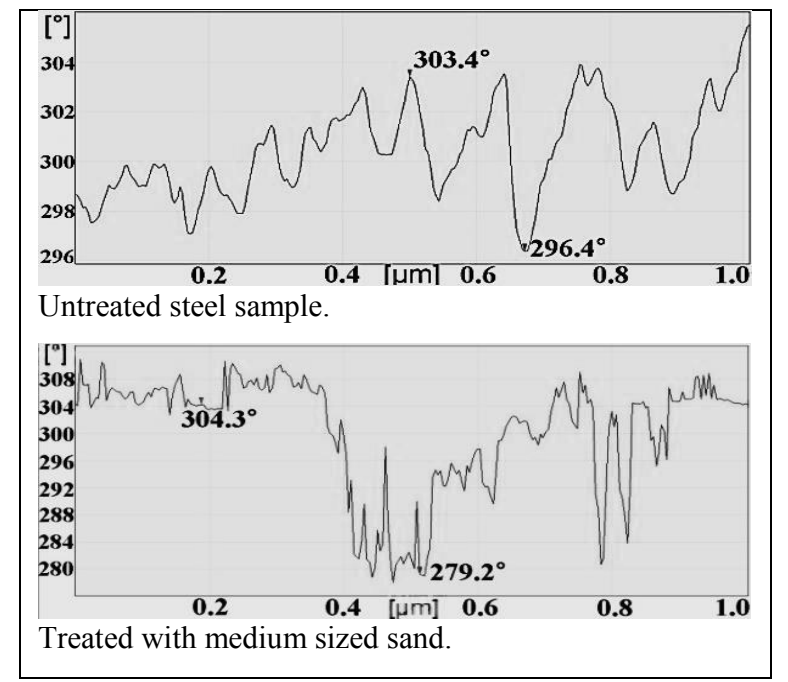

Fig. 3. USPM cross-section profiles of treated and untreated steel samples.

At small velocity and impact angle, the erodent particles exhibit angular slip at the target surface. Thus, impacting force vector splits into horizontal and vertical components. At small impact angle, axial component is the major contributor to the energy transfer from the sand stream to the exposed surface. It reveals that the force, exerted by the sand particles on the exposed surface, was small and so the surface damages. Also, if the impingement angle is small, the collisions among the incoming and rebounding sand particles would appreciably reduce the kinetic energy of the particles in the sand stream $[9,10]$. This interference acts as 
a barrier to prevent the incident particles impacting on the sample surface. This leads to a reduced erosion rate for the angles ranging from $45^{\circ}$, as shown in Figure 4.

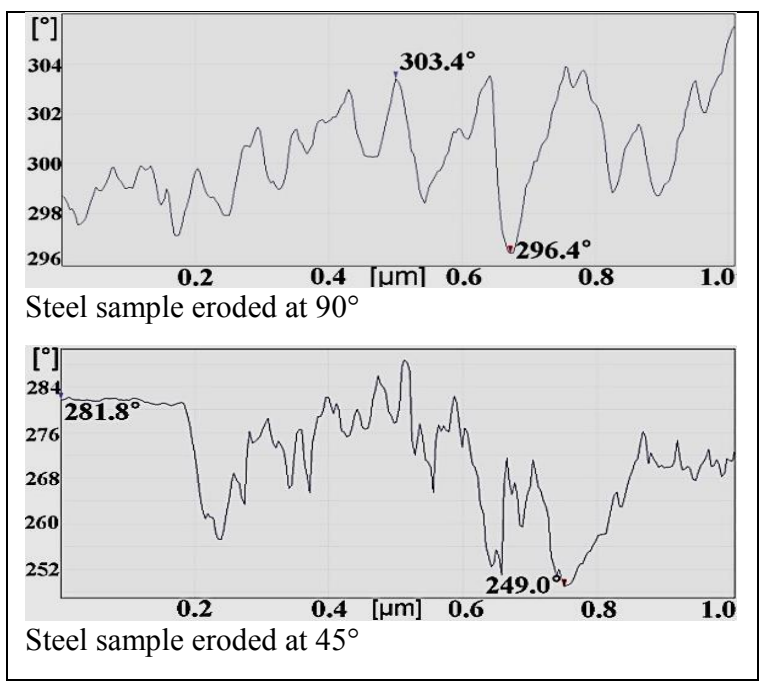

Fig. 4. USPM cross-section profiles obtained at different impact angles.

Figure 5 shows USPM cross-section profiles at different gas flow velocities. It was observed that the erosion effect of $72 \mathrm{ft} / \mathrm{s}$ gas flow velocity is more severe as compared to $54 \mathrm{ft} / \mathrm{s}$. The craters in USPM profiles obtained with $72 \mathrm{ft} / \mathrm{s}$ velocity were wider compared to the craters in the profiles obtained with $54 \mathrm{ft} / \mathrm{s}$ velocity.

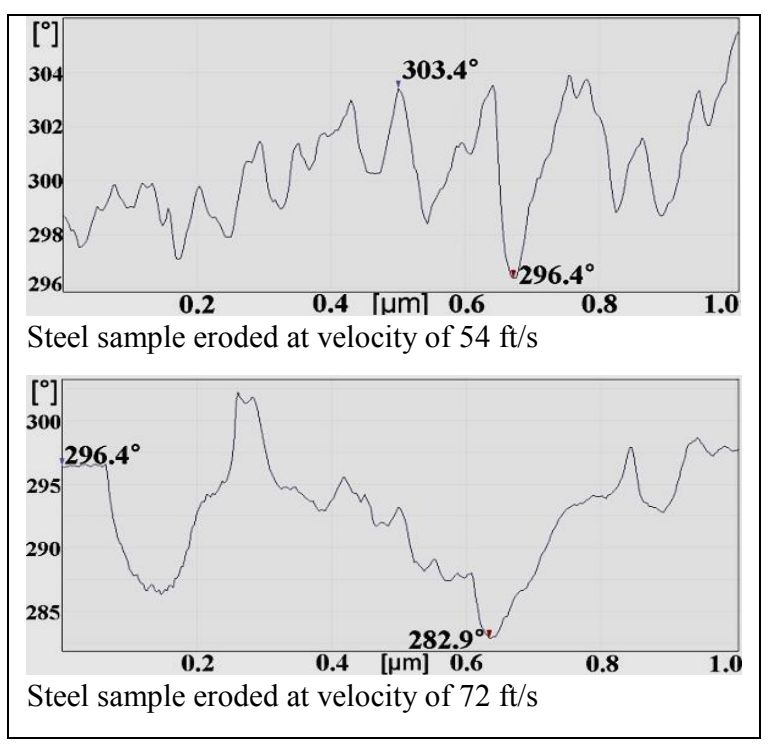

Fig. 5. USPM cross-section obtained at different flow velocities.

In fact, the deepest crater in erosion profiles, obtained with sand stream velocity of 72 $\mathrm{ft} / \mathrm{s}$, was measured about $13^{\circ}$. On the other hand, the craters in erosion profiles, obtained with $54 \mathrm{ft} / \mathrm{s}$, only yielded around $7^{\circ}$ tip difference with the normal plane. When particle velocity is low, only fewer sand particles would have the required kinetic energy for plastic deformation of the target surface. majority of the erodent particles exhibited energies lower 
than the threshold value, which only contribute to the elastic deformation of the target surface. However, the number of particles, reaching the critical energy, increase with an increase in stream velocity. Such particles may cause deformation and removal of the material from the exposed surface.

Figure 6 shows USPM cross-section profiles of steel samples place at a distance of $10 \mathrm{~mm}$ and $20 \mathrm{~mm}$ from the nozzle. By comparing the results, it was observed that the erosion pattern for $20 \mathrm{~mm}$ nozzle distance consists of craters smaller than that of erosion for $10 \mathrm{~mm}$ nozzle distance. These craters were widely spread on the target surface. While the craters in profiles obtained at $10 \mathrm{~mm}$ nozzle distance were not widely spread, the erosions areas were narrow and concentrated as compared to the areas treated at a distance of $20 \mathrm{~mm}$.

By referring to the cross-sectional profiles, the craters at $20 \mathrm{~mm}$ distance were not as deep as the craters observed at $10 \mathrm{~mm}$, in which the deepest is only $3.9^{\circ}$. In addition, the patterns for $20 \mathrm{~mm}$ nozzle distance revealed lot many small-sized rough areas as compared to the patterns for $10 \mathrm{~mm}$ nozzle distance [9].

It is concluded that the erosion severity of nozzle placed at a distance of $10 \mathrm{~mm}$ is higher than that at $20 \mathrm{~mm}$. Shorter distance will show shorter impact time, causing higher impact force on the surface of the target plate. On the other hand, $20 \mathrm{~mm}$ distance between the nozzle and the target causes the particle's momentum to drop slightly due to energy loss during the travel of sand to the metal surface. In addition, the longer distance will also cause the impact region on the surface plate to become bigger, indicating that the sand-contained air flow becomes unstable and raises the impact radius, thus lowering the impact energy. Herein, the bigger impact radius will cause the energy-momentum to be less concentrated.

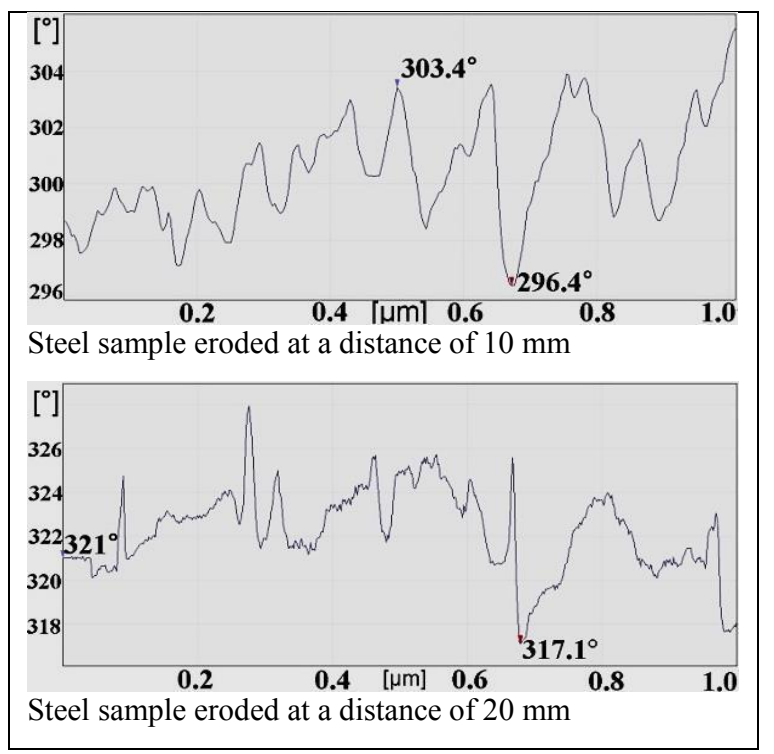

Fig. 6. USPM cross-section profiles obtained at different nozzle positions.

\section{Conclusions}

This study concludes that sand blasting significantly altered the surface morphology and composition of the stainless-steel coupons. At high velocity and small target distance, the sand particles diffused into the metal body. Microscopic examination of the eroded coupons showed that both the impact velocity and angle contribute to the erosion of the production flow-lines. However, impact angle plays dominating role in damaging the flow-lines due to 
sanding. The erosion due to sanding can be minimized by reducing the sharp edges and bends in the flow-line. An impact angle of $45^{\circ}$ causes highest erosion of the target surface. In line with this, the highly accelerated particles erode the target surface more adversely than those imping the target at lower velocities. Apart from the size and angle, the shorter nozzle distance showed more severe erosion as compared to the longer nozzle distances.

\section{References}

1. B. Gandhi, S. Borse, Ind. J. Eng. Mater. Sci., 9, 480 (2002)

2. C. Fan, B.S. McLaury, S.A. Shirazi, E.F. Rybicki, NACE International Annual Conference, Salt Lake City, Utah, USA, (2012)

3. Y. Garsany, D. Pletcher, D. Sidorin, W.M Hedges, Corrosion, 60, 1155 (2004)

4. M.A. Islam, Z.N. Farhat, Wear, 311, 180 (2014)

5. S. Shirazi, B. McLaury, J. Shadley, E. Rybicki, J. Pet Technol., 47, 693 (1995)

6. C.Y. Wong, C.B. Solnordal, J. Wu, SPE Production \& Operations, 29, 61 (2013)

7. D. Celotta, U. Qureshi, E. Stepanov, D. Goulet, J. Hunter, C. Buckberry, R. Wood, Wear, 263, 278 (2007)

8. M.Y. Naz, N.I. Ismail, S.A. Sulaiman, S. Shukrullah, Surf. Rev. Lett., 23, 1650035 (2016)

9. M. Lemistre, D. Soulevant, F. Micheli, A. Déom, Wear, 233, 712 (1999)

10. M.Y. Naz, N.I. Ismail, S.A. Sulaiman, S. Shukrullah, Sci. Rep., 5, 16583 (2015) 